\title{
Los Tipos Craneométricos Caninos Aparecen Bien Expresados a Nivel de Conformación del Arco Cigomático
}

\author{
Craneometric Canine Types are Well Expressed at \\ the Level of the Zygomatic Arch Conformation
}

Vedat Onar $^{1}$; Abu Bakar Siddiq ${ }^{2}$, Rahmi Asal ${ }^{3}$ \& Pere M. Parés-Casanova ${ }^{4}$

ONAR, V.; SIDDIQ, A. B.; ASAL, R. \&PARÉS-CASANOVA, P. M. Los tipos craneométricos caninos aparecen bien expresados a nivel de conformación del arco cigomático. Int. J. Morphol., 38(1):78-82, 2020.

RESUMEN: Dentro del espectro de conformación del cráneo, se reconocen generalmente tres amplias categorías que se corresponden con el concepto de biotipo cefálico, determinado por el Índice Cefálico. El Estos tres biotipos cefálicos son: el braquiocefálico, mesaticefálico y dolicocefálico, pero están basados en medidas lineales. A fin de revisar esta clasificación en base a su geometría, se estudiaron 53 cráneos de perros adultos, correspondientes a los tres grupos craneométricos descritos: 16 braquicéfalos, 20 mesaticéfalos y 17 dolicocéfalos. Para ello se obtuvieron fotografías en el plano ventral, en las que posteriormente se ubicaron 17 hitos anatómicos que se analizaron mediante técnicas de morfometría geométrica. De estos hitos, 5 correspondían a la zona neurocraneal y el resto al esplacnocráneo. Los tres grupos craneométricos mostraron diferencias estadísticamente significativas entre ellos tanto por el tamaño como por la forma. Las variables que contribuyeron más a explicar la diferenciación fueron las ubicadas en el margen más lateral de los arcos cigomáticos y en la base de este mismo arco. Las variables esplacnocraneales presentaban una alometría mucho más marcada que las neurocráneos. Puesto que el arco cigomático debe ser considerado como parte del esplacnocráneo, sugerimos que es tan importante el índice cefálico (que tiene en cuenta la máxima anchura de la cabeza) como el facial (que tiene en cuenta la máxima anchura de la cara). La conformación neurocraneal sería mucho más conservativa y por ende el índice craneal, de mucho menor poder discriminatorio entre grupos. El cambio entre tipos se debería a los músculos masetero y temporal, que tienen su inserción en el arco.

PALABRAS CLAVE: Canis familiaris; Cráneo; Indice cefálico; Indice craneal; Mesaticefálico.

\section{INTRODUCCIÓN}

Dentro del espectro de conformación del cráneo, se reconocen generalmente tres amplias categorías que se corresponden con el concepto de biotipo cefálico, determinado por el Índice Cefálico. Este índice ya fue usado con fines antropológicos por el anatomista Retzius, en 1840 (Lakshmi Kumari et al., 2015) y se calcula como la relación entre la anchura máxima y la longitud máxima de la cabeza (Lakshmi Kumari et al.). Los tres biotipos cefálicos actualmente reconocidos en base a este Índice son: el braquiocefálico, el mesaticefálico y el dolicocefálico (Aparicio, 1944) (Sisson \& Grossman, 1985; Evans \& de Lahunta, 2013; Teng et al., 2016):

1) Dolicocéfalos (del griego antiguo kephalê, "cabeza" y dolikhos, "largo y delgado") en donde la cabeza es estrecha y larga.
2) Braquiocefálico (del griego antiguo kephalê, "cabeza" y brakhys, "corto") en donde la cabeza es corta y ancha.

3) Mesaticefálico (del griego antiguo kephalê, "cabeza" y mesos, "medio"), de forma intermedia a las anteriores.

El Índice Cefálico, que se refiere a la cabeza en conjunto, representa la base para todas las razas caninas existentes, pero existen igualmente índices alternativos que también han sido utilizados para clasificar las razas caninas: índices facial, palatino, nasal, craneal, etc. (Lakshmi Kumari et al.; Igado, 2017). Todos ellos se basan en la relación entre un largo y un ancho (Teng et al.). Las fórmulas más aceptadas, conocidas e históricas son los índices Stockard y Evans. Una excelente revisión de todos los índices sugeridos por los diferentes autores ha sido realizada por Onar et al. (2012). Aunque en el pasado se le dio un valor excesivo a los índi-

\footnotetext{
${ }^{1}$ Department of Anatomy, Faculty of Veterinary Medicine, Istanbul University-Cerrahpasa, 34320 Avcılar, Istanbul, Turkey. E-mail: onar@istanbul.edu.tr ${ }^{2}$ Department of Anthropology, Faculty of Letters, Mardin Artuklu University, 47200 Artuklu, Mardin, Turkey. E-mail: abubakarsiddiq@artuklu.edu.tr ${ }^{3}$ Istanbul Archaeological Museum, Osman Hamdi Bey Yokusu, Gülhane, 34122 - Fatih, Istanbul, Turkey. E-mail: rahmiasal@hotmail.com ${ }^{4}$ Department of Animal Science, University of Lleida. Av. Rovira Roure, 191, 25198 Lleida, Catalonia, Spain. E-mail:peremiquelp@ca.udl.cat
} 
ces cefálicos en la actualidad se sigue apreciando su utilidad, como la de cualquier otro carácter.

Si consideramos que la forma es más relevante que el tamaño en los análisis taxonómicos y de anatomía funcional, y puesto que el tamaño tiene una mayor variabilidad entre grupos, estudiar los cráneos por métodos tradicionales puede enmascarar la variación de forma. A diferencia de las variables lineales, que son las que estudian esos métodos tradicionales, un conjunto de coordenadas cartesianas, que son las variables que estudia la morfometría geométrica, no sólo tiene información sobre el tamaño y la forma de los objetos, sino también sobre la posición y la orientación relativa de las coordenadas. Así, la forma puede ser definida como la información presente en un conjunto de coordenadas después que la localización, la escala y la orientación son excluidas (Zelditch et al., 2004). Es importante notar que el tamaño de una estructura puede expresarse como un valor absoluto; la forma en cambio, sólo puede expresarse en términos relativos (Adams et al., 2013).

\section{MATERIAL Y MÉTODO}

Muestra. Se analizó una muestra de 53 cráneos de perros adultos, con dentadura permanente, correspondientes a los tres grupos craneométricos descritos: 16 de ellos correspondientes a razas braquicéfalas (7 Boxer, 4 Bulldog, 2 Pequinés, 1 Cow Chow, 1 Sharpei y 1 Pitbull), 20 mesaticéfalas (2 Pointer, 1 Pomeraniano, 1 Husky y 16 del período Bizantino) y 17 dolicocéfalas (10 Pastor Alemán, 6 Dobermann y 1 Collie). Estos especímenes, recolectados con anterioridad para otros usos, se hallan depositados en el Laboratorio de Osteoarqueología de la Facultad de Medicina Veterinaria de Istambul, en Turquía. No se consideraron para este estudio cráneos con evidencias claras de patologías ni ejemplares jóvenes.

Se obtuvieron imágenes digitales, en la cara ventral, de cada cráneo, mediante una cámara digital Nikon D1500 equipada con una lente $40 \mathrm{~mm}$ 1:2.8G MicroNikkor. El plano ventral estaba orientado lo más paralela a la cámara, a fin de eliminar distorsiones de las posiciones relativas de los ángulos debido a un efecto parallax, y la cámara situándose a su vez a suficiente distancia para asegurar que el cráneo ocupase únicamente una parte del campo visual, libre de distorsiones. Se incluía en cada toma un patrón milimétrico. Las imágenes eran almacenadas en extensión jpg de unos 9 $\mathrm{Mb}$ y posteriormente transferidas al computador.

Hitos anatómicos y digitalización. En cada imagen se ubicaron posteriormente, con el programa tpsDig v. 1.40 (Rohlf,
2015), 17 hitos anatómicos, 14 pareados y 3 de impares (Fig. 1) y se obtuvo un archivo de extensión .tps con las coordenadas cartesianas. De estos hitos, 5 correspondían a la zona neurocraneal y el resto al esplacnocráneo. Se realizaron dos réplicas de esta digitalización a fin de establecer el error. Esta doble réplica la realizó el primer autor (PMPC). Con las coordenadas cartesianas se procedió seguidamente a una superposición Procrustes clásica -que busca minimizar la suma de las diferencias al cuadrado entre todos los pares de hitos-. El tamaño se valoró como el tamaño de centroide equivalente a la raíz cuadrada de la suma de las distancias al cuadrado de cada hito desde el centroide- (Zelditch et al.). La conveniencia de utilizar el tamaño del centroide como variable de tamaño radica en que es la única alternativa que no se correlaciona con la forma cuando el modelo nulo de alometría es verdadero (Adams et al.). Las distancias Procrustes entre especímenes se computaron como distancias euclideanas.

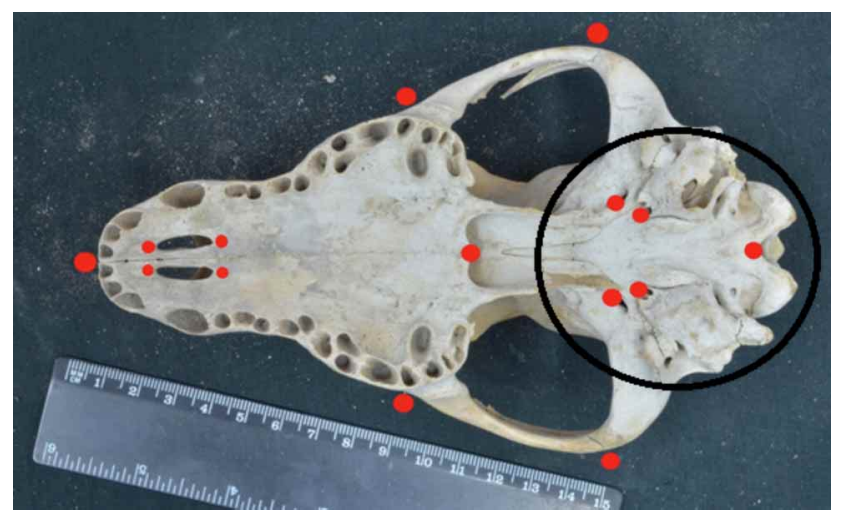

Fig. 1. Hitos anatómicos usados en este estudio de morfometría geométrica, 14 de ellos pareados y 3 en el plano medio. El círculo engloba los 5 hitos anatómicos del neurocráneo. El resto pertenecerían al esplacnocráneo.

Análisis estadístico. Se analizaron los datos (coordenadas Procrustes) mediante un ANOVA, y seguidamente mediante un Análisis de Componentes Principales (ACP) a partir de la matriz de varianza-covarianza, mediante el cual se exploraron los principales ejes de variación morfológica en el espacio tangente, para observar las tendencias de variación entre y dentro de las muestras. Se realizó también una regresión utilizando el tamaño de centroide como variable independiente y las coordenadas Procrustes logarítmicamente transformadas- como dependientes. Finalmente, se realizó un Análisis Canónico con un ajuste "jacknifed" para corregir el sesgo de estimación.

Los tratamientos estadísticos se realizaron con el programa MorphJ v. 1.06c (Klingenberg, 2011), excepto el Análisis Canónico, que se realizó con el programa PAST v. 2.17c (Hammer et al., 2001). 
Consideraciones éticas: Los cráneos procedían de una colección ya existente, por lo que al no haberse procedido al sacrificio de ningún animal no se consideró necesaria ninguna autorización ética.

\section{RESULTADOS}

No aparecieron diferencias estadísticamente significativas entre réplicas, con un mero $0,006 \%$ de variación debida al error, por lo que los análisis ulteriores se realizaron con los valores individuales promediados. Los tres grupos craneométricos mostraron diferencias estadísticamente significativas entre ellos tanto por el tamaño $(\mathrm{F}=21,53$;

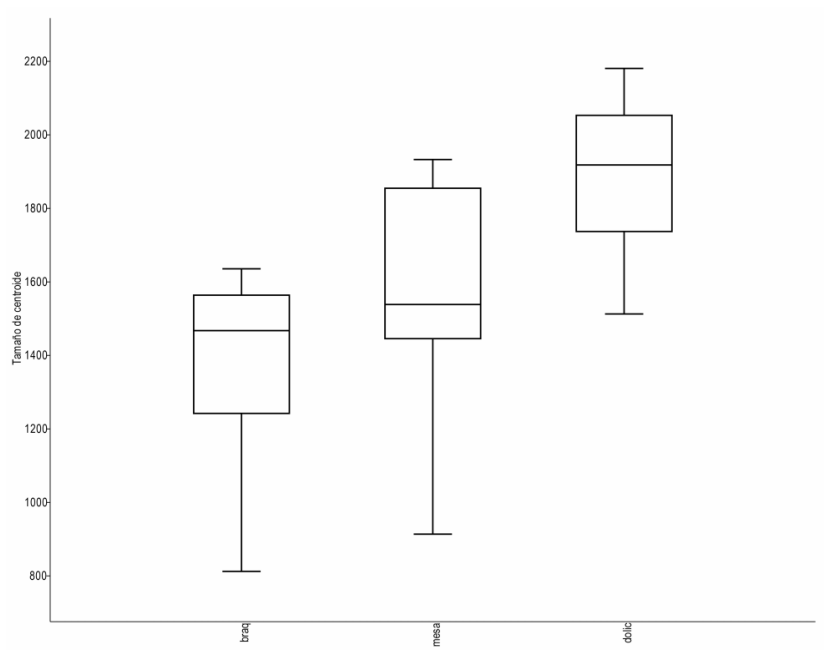

Fig. 2. Diagramas de cajón de los tamaños de centroide para los 3 tipos craneométricos estudiados (16 de ellos correspondientes a razas braquicéfalas, "braq", 20 mesaticéfalas, "mes" y 17 dolicocéfalas, "dolic"). Aparecieron diferencias estadísticamente significativas entre ellos por el tamaño $(F=21,53$; $p<0,0001)$ $\mathrm{p}<0,0001)$ (Fig. 2) como por la forma $(\mathrm{F}=298,43 ; \mathrm{p}<0,0001)$. El ACP mostró que un $61,58 \%$ de la varianza total observada era explicada en el Componente Principal $1(\mathrm{CP} 1)$ y un $16,30 \%$, por el CP2 (CP1+CP2 $=77,89 \%$ ) (Fig. 3). Las variables que contribuyeron más a explicar esta varianza $(>[0,3])$ fueron las del eje Y ubicadas en el margen más lateral de los arcos cigomáticos y en la base de este mismo arco (Fig. 4). De hecho, si se vuelven a comparar los tres grupos atendiendo únicamente estos cuatro hitos ubicados en el arco cigomático, siguen apareciendo diferencias significativas entre ellos $\mathrm{p}<0,0001)$. Por lo que se refiere a la regresión, un $31,68 \%$ de la variación en la forma se explicó por la variación en el tamaño, de lo que se infiere una presencia acusada de alometría craneal $(\mathrm{p}=0,01)$. Esta alometría es mucho más importante en el esplacnocráneo $(29,3 \%)$ que en el neurocráneo (16,2 \%). Finalmente, el Análisis Canónico recurriendo únicamente a estos 4 hitos, mostró un notable poder de discriminación, oscilando del 58,8 \% para los dolicocéfalos al $85 \%$ para los mesaticéfalos (Tabla I).

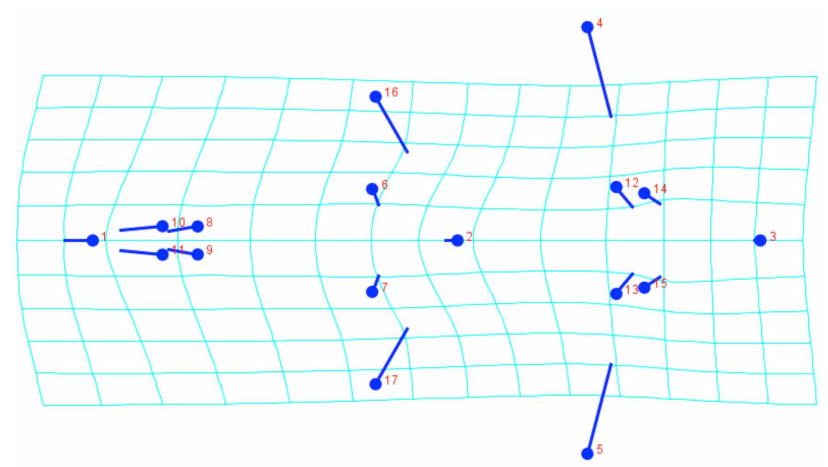

Fig. 4. Rejilla de deformación para el componente principal 1, que explicó el 61,58 \% de la varianza total observada. Las variables que contribuyeron más a explicar esta varianza fueron las del eje Y ubicadas en el margen más lateral de los arcos cigomáticos (4 y 5) y en la base de este mismo arco (16 y 17).

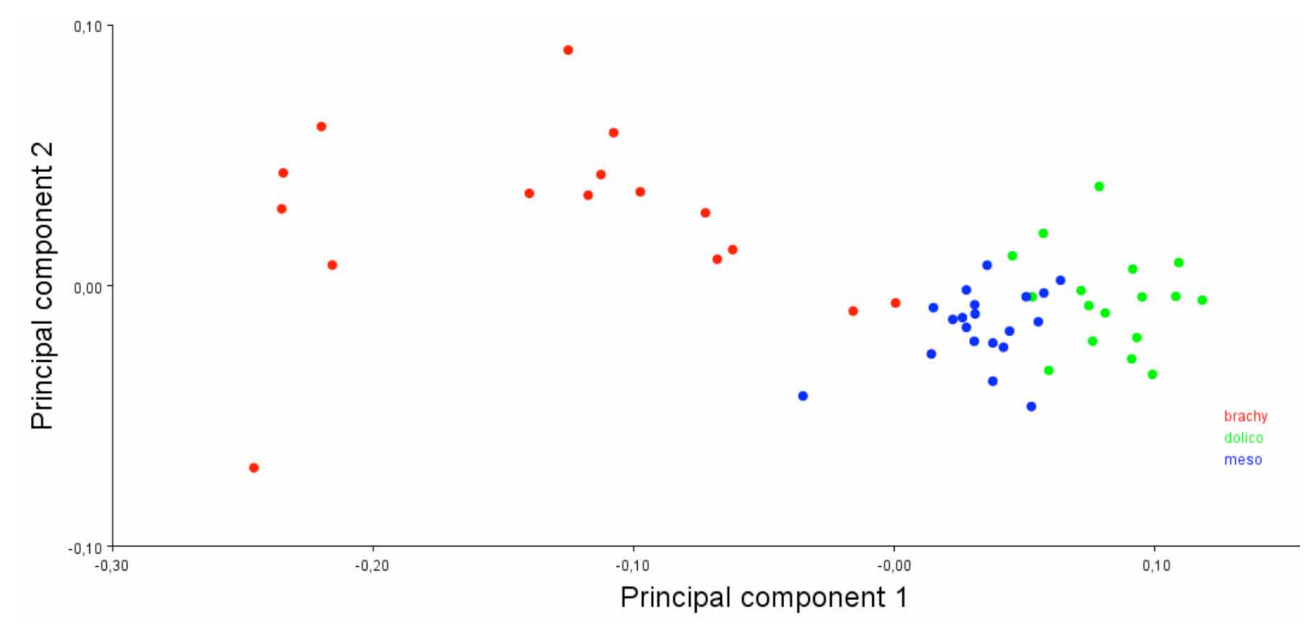

Fig. 3. Análisis de componentes principales para los dos primeros componentes para los 3 tipos craneométricos estudiados (16 de ellos correspondientes a razas braquicéfalas, "brachy", 20 mesaticéfalas, "meso" y 17 dolicocéfalas, "dolico"). El ACP mostró que un 61,58 \% de la varianza total observada era explicada en el Componente principal 1 , y un 16,30 $\%$, por el Componente Principal $(\mathrm{CP} 1+\mathrm{CP} 2=77,89 \%)$. 
Tabla I. Resultados del Análisis Discriminante utilizando únicamente los 4 hitos ubicados en el arco cigomático para los 3 tipos craneométricos estudiados (16 de ellos correspondientes a razas braquicéfalas, 20 mesaticéfalas y 17 dolicocéfalas). Apareció un aceptable poder de discriminación, oscilando del 58,8 \% para los dolicocéfalos al $85 \%$ para los mesaticéfalos.

\begin{tabular}{lllll}
\hline & Braquicéfalo & Mesaticéfalo & Dolicocéfalo & TOTAL \\
\hline Braquicéfalo & 13 & 1 & 2 & 16 \\
Mesaticéfalo & 0 & 17 & 3 & 20 \\
Dolicocéfalo & 0 & 7 & 10 & 17 \\
\hline
\end{tabular}

\section{DISCUSIÓN}

Los estudios morfométricos en cráneo de perro suelen haberse basado en el empleo de medidas lineales entre puntos anatómicos, y analizados mediante técnicas estadísticas uni y multivariadas (enfoque denominado morfometría tradicional). El enfoque cuantitativo (morfometría geométrica o síntesis morfométrica) permite obtener variables de tamaño y de forma a partir de las coordenadas cartesianas de puntos anatómicos y analizarlas posteriormente mediante el empleo de técnicas estadísticas multivariadas (Adams et al.).

De nuestro estudio se desprende que a pesar de la infinidad de fórmulas y propuestas para agrupar las razas caninas en base a su tipología craneal, seran básicamente la anchura y la base del arco cigomático las que van a determinar más la pertinencia a un grupo cefálico u a otro -dolicocéfalo, mesaticéfalo o braquicéfalo-. Así como, por ejemplo, la cabeza de un perro dolicocéfalo presenta siempre una cresta sagital externa destacada (Dyce et al., 1999), los arcos cigomáticos presentarían igualmente formas diferentes en cada biotipo craneométrico, formas expresadas en su anchura y en el ángulo del hueso cigomático a nivel rostral, cuando se une a la parte facial. Este cambio en el arco se explicaría por un cambio en la funcionalidad masticatoria, puesto que es punto de inserción de importantes músculos masticatorios (masetero y temporal) (Barone, 2000), cuya función es indudable que varía entre tipos craneométricos y, por ende, sus puntos de ancoraje diferentes, con el consiguiente cambio en la conformación ósea.

Pero, a la vista de la diferencia de tamaño craneal entre grupos, y por el comportamiento alométrico de la forma -la dolicocefalización se explicaría por un mayor tamaño de la cabeza y la braquicefalia por tallas menores-, las variables lineales que puedan obtenerse en los arcos cigomáticos deberían relativizarse en base a la longitud total del cráneo, o sea, que va a ser importante la relación porcentual de este medidas, o lo que es lo mismo, va a ser importante un índice bidimensional (Evans \& de Lahunta).
Puesto que cualquier punto sobre el arco cigomática debe ser considerada como medida tomada sobre el esplacnocráneo (Lakshmi Kumari et al.), sugerimos que sean tan importantes el índice cefálico (que tiene en cuenta la longitud total de la cabeza) como el facial (que tiene en cuenta la máxima anchura de la cara a nivel del arco cigomático) (Lakshmi Kumari et al.). De hecho, la diferenciación entre las cabezas de las distintas razas de perro residen sobre todo en la distinta longitud que puede alcanzar la región facial, lo que afirman ya otros autores (Dyce et al.; Evans \& de Lahunta), lo que reafirma la importancia del índice facial. La conformación neurocraneal, correspondiente a la bóveda craneal, sería mucho más conservativa y por ende el índice craneal, presentaría un menor poder discriminatorio o, en otras palabras, el carácter del índice craneal, mucho más relativo que el cefálico y el facial. El índice craneofacial, que se calcula como el cociente entre la longitud del neurocráneo y del esplacnocráneo (Dyce et al.), podría igualmente resultar revelador.

Los resultados descritos en este trabajo, demuestran de todos modos que la morfometría geométrica del arco cigomático es una herramienta idónea en la discriminación de los tipos cefálicos en el perro, siendo un método de fácil aplicación y con validación estadística. En este sentido, resulta interesante que utilizando cuatro puntos anatómicos en el arco cigomático, se logren diferenciar esos distintos tipos. Sin embargo, aún es necesario realizar análisis adicionales que incluyan otras razas, con el fin de evaluar la relevancia de los puntos anatómicos seleccionados.

Los resultados de nuestro estudio sugieren además que la morfometría geométrica es particularmente apropiada para el análisis fenotípico de variación de la forma del arco cigomático. Esta herramienta, la morfometría geométrica, permite descomponer la variación resultante de la fisiología de los individuos (variación de tamaño) de aquellas más estables propias de la población, producto del componente genético (variación de la forma), o sea, que los 3 
biotipos craneométricos de los perros se confirman mediante el uso de la información contenida en la conformación (forma) del arco cigomático. Por otro lado, esta investigación abre camino en estudios de especies salvajes de cánidos.

\section{AGRADECIMIENTOS}

A los árbitros anónimos por los valiosos comentarios y sugerencias en mejora de la versión final del presente trabajo.

ONAR, V.; SIDDIQ, A. B.; ASAL, R. \&PARÉS-CASANOVA, P. M. Craneometric canine types are well expressed at the level of the zygomatic arch conformation. Int. J. Morphol., 38(1):78-82, 2020.

SUMMARY: Within the wide conformation of skull spectrum, there are generally three recognized broad categories that correspond to the concept of cephalic biotype, determined by the cephalic index. The three cephalic biotypes are: brachiocephalic, mesaticephalic and dolichocephalic, which are based on linear measures. In order to revise this classification based on its geometry, we studied 53 skulls of adult dogs, corresponding to the three craneometric groups previously described: 16 brachycephalic, 20 mesaticephalic and 17 dolichocephalic. Images on ventral plane were obtained and 17 anatomical landmarks were subsequently located and analyzed by means of geometric morphometric techniques. Five of those landmarks corresponded to the neurocraneal area and the rest of the splanchnocranium. The three craneometric groups showed statistically significant differences between them for both size and shape. The variables that contributed to the differentiation between them were located along the edge of the zygomatic arches and on the basis of this arch. Splanchnocranial variables also presented a much more marked allometry than the neurocraneal variables. Since the zygomatic arch should be considered as part of the splanchnocranium, we suggest that the cephalic index (which takes into account the maximum width of the head) is as important as the facial index (which takes into account the maximum width of the face). The neurocraneal index would be much more conservative, and therefore less discriminatory between the groups.

KEY WORDS: Canis familiaris; Cranium; Cranial index; Cephalic index; Mesaticephalic.

\section{REFERENCIAS BIBLIOGRÁFICAS}

Adams, D. C.; Rohlf, F. J. \& Slice, D. E. A field comes of age: geometric morphometrics in the 21st century. Hystrix It. J. Mamm., 24(1):7-14, 2013.

Aparicio, G. Zootecnia Especial. Etnología Compendiada. $3^{\mathrm{a}}$ ed. Córdoba, Imprenta Moderna, 1944.

Barone, R. Anatomie Comparée des Mammifères Domestiques. Tome 2. Artrhologie et Myologie. Paris, Vigot, 2000.
Dyce, K. M.; Sack, W. O. \& Wensig, C. J. G. Anatomía veterinaria. México, Manual Moderno, 1999.

Evans, H. E. \& de Lahunta, A. Miller's Anatomy of the dog. St. Louis (Mo), Elsevier Saunders, 2013.

Hammer, Ø.; Harper, D. A. T. \& Ryan, P. D. PAST v. 2.17c. Palaeontol. Electron., 4(1):1-229, 2001.

Igado, O. O. Skull typology and morphometrics of the nigerian local dog (Canis lupus familiaris). Niger. J. Physiol. Sci., 32(2):153-8, 2017.

Klingenberg, C. P. MorphoJ: an integrated software package for geometric morphometrics. Mol. Ecol. Resour., 11(2):353-7, 2011.

Lakshmi Kumari, K.; Vijaya Babu, P. V. S. S.; Kusuma Kumari, P. \& Nagamani, M. A study of cephalic index and facial index in Visakhapatnam, Andhra Pradesh, India. Int. J. Res. Med. Sci., 3(3):6568, 2015.

Onar, V.; Çakirlar, C.; Janeczek, M. \& Kiziltan, Z. Skull typology of Byzantine dogs from the Theodosius Harbour at Yenikapi, Istanbul. Anat. Histol. Embryol., 41(5):341-52, 2012.

Rohlf, F. J. The tps series of software. Hystrix It. J. Mamm., 26(1):9-12, 2015.

Sisson, S. \& Grossman, J. D. Anatomía de los animales domésticos. Tomo II. Barcelona, Salvat, 1985.

Teng, K. T.; McGreevy, P. D.; Toribio, J. A. L. M. L. \& Dhand, N. K. Trends in popularity of some morphological traits of purebred dogs in Australia. Canine Genet. Epidemiol., 3:2, 2016.

Zelditch, M. L.; Swiderski, D. L. \& Sheets, H. D. Geometric Morphometrics for Biologists: A Primer. Boston (MA), Elsevier Academic Press, 2004.

Corresponding author:

Pere M. Parés-Casanova PhD

Department of Animal Science School of Agrifood and

Forestry

Science and Engineering University of Lleida

Av. Rovira Roure 191

Catalunya

SPAIN

Email: peremiquelp@ca.udl.cat

Received: 05-06-2019

Accepted: 29-07-2019 